\title{
Images
}

\section{Hammer time: penetrating trauma from sledgehammer use}

\author{
Daniel K. Ting, MD*; Anthony Kwan, MD*
}

A healthy 24-year-old landscaper was using a sledgehammer and steel wedge to split rock. After a strike of the hammer, he felt pain in his abdomen and noticed a small laceration to the epigastric region. He also recognized an incongruity in the sledgehammer head.

En route to the emergency department, the patient had one instance of nonbloody emesis. He presented with normal vital signs but developed epigastric pain and suffered multiple episodes of hematemesis. On exam he had a $7-\mathrm{mm}$ laceration of the right anterior abdominal wall and signs of peritonitis. Both a radiograph (Figure 1) and computed tomography (CT) scan (Figure 2) of the abdomen were obtained.

The patient received tranexamic acid, ondansetron, and antibiotics. General surgery performed an urgent laparotomy, during which one litre of blood was evacuated from the peritoneal cavity, and an anterior stomach wall defect

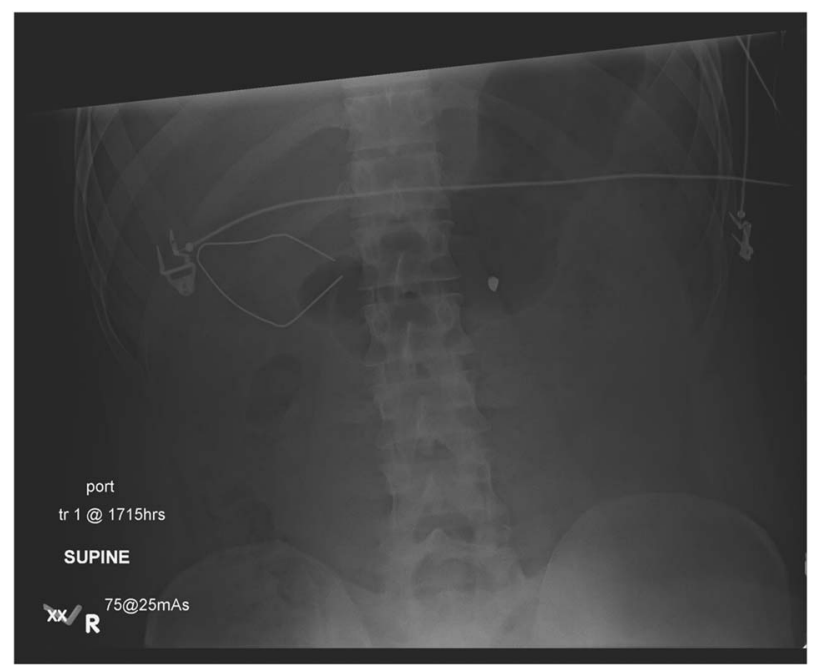

Figure 1. Portable frontal radiograph showing a radiodense foreign body in the left upper abdomen. The location of the wound is between the open ends of the paper clip. was repaired. No foreign body was found. The patient was discharged home in stable condition 4 days later.

Penetrating trauma from sledgehammer use has been reported and can be fatal. ${ }^{1-3}$ The resulting fragments are estimated to reach velocities of 110 metres per second, ${ }^{4}$ which is on the same order of magnitude as a bullet and can present similarly to a gunshot wound. The physical examination of the wound can be unimpressive. A key in management is recognizing that a penetrating foreign body may cause significant organ injury, even if the patient appears initially stable.

Keywords: abdominal injuries, emergency medicine, foreign bodies, penetrating wounds, wounds and injuries

Competing interests: None declared.

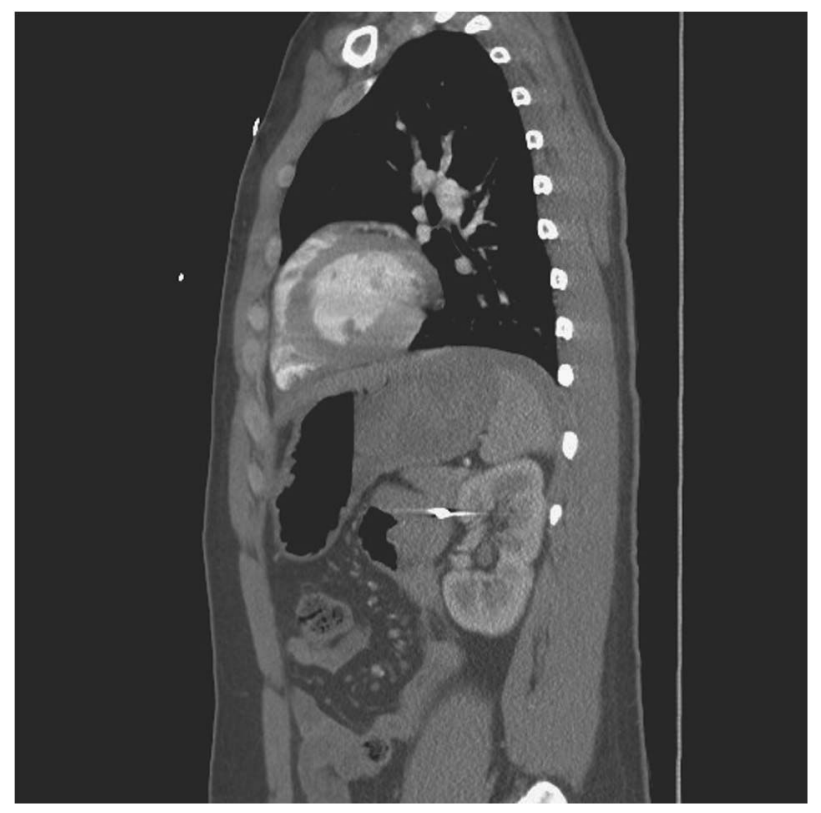

Figure 2. Sagittal CT slice shows the foreign body lodged in the retroperitoneum, anterior to the left kidney and inferior to the pancreatic tail.

From the *Department of Emergency Medicine, University of British Columbia, Kelowna, BC.

Correspondence to: Dr. Daniel K. Ting, Department of Emergency Medicine, Kelowna General Hospital, 2312 Pandosy Street, Kelowna, BC V1Y 1T3; Email: daniel.ting@alumni.ubc.ca 


\section{REFERENCES}

1. Smith ME, Zumwalt R. Occupational deaths due to penetrating chest injuries from sledgehammer fragments. $A m \mathcal{F}$ Forensic Med Pathol 2004;25(1):71-3.

2. Lipscomb HJ, Schoenfisch AL. Nonfatal tool- or equipmentrelated injuries treated in US emergency departments among workers in the construction industry, 1998-2005. Am 7 Ind Med 2010;53(6):581-7.

3. Turkcuoglu P, Aydogan S. Intracranial foreign body in a globe-perforating injury. Can 7 Ophthalmol 2006;41(4):504-5.

4. McIntire HO, Manning GK. A study of the spalling of hand hammers. Battelle Memorial Institute, unpublished report; 19 May 1958. 